\title{
A rapidly variable narrow $X$-ray iron line in Mkn 841
}

\author{
P. O. Petrucci ${ }^{1}$, G. Henri ${ }^{1}$, L. Maraschi ${ }^{2}$, P. Ferrando ${ }^{3}$, G. Matt ${ }^{4}$, M. Mouchet $^{5}$, C. Perola ${ }^{4}$, S. Collin ${ }^{5}$, \\ A. M. Dumont ${ }^{5}$, F. Haardt ${ }^{7}$, and L. Koch-Miramond ${ }^{3}$ \\ 1 Laboratoire d'Astrophysique de Grenoble, BP 43, 38041 Grenoble Cedex 9, France \\ 2 Osservatorio Astronomico di Brera, via Brera 28, 02121 Milano, Italy \\ 3 Service d'Astrophysique, DSM/DAPNIA/SAp, CE Saclay, 91191 Gif-sur-Yvette Cedex, France \\ 4 Dipartimento di Fisica, Università degli Studi "Roma tre", via della Vasca Navale 84, 00046 Roma, Italy \\ ${ }^{5}$ LUTH, Observatoire de Paris, Section de Meudon, 92195 Meudon Cedex, France \\ 6 Istituto di Astrofisica Spaziale, CNR, via Fosso del Cavaliere 100, 00133 Roma, Italy \\ 7 Università dell'Insubria, via Lucini 3, 22100 Como, Italy
}

Received 26 February 2002 / Accepted 5 April 2002

\begin{abstract}
We report on the detection of a rapidly variable narrow Fe K $\alpha$ line in Mkn 841. The source has been observed two times by XMM-Newton and simultaneously with BeppoSAX. The two observations, of about $10 \mathrm{ks}$ long each, were separated by $\sim 15$ hours. The line flux reaches a maximum during the first observation and is significantly reduced in the second one. The continuum shape and flux, instead, keep roughly constant between the two pointings. Such rapid variability of a narrow (unresolved by the XMM-pn instrument) line has never been reported in the past. These results are not easily explained in the standard cold reflection model where the narrow line component is supposed to be produced far from the primary X-ray source (e.g. from the torus) and is thus not expected to vary rapidly. Different interpretations are discussed.
\end{abstract}

Key words. galaxies: individual: Mkn 841 - galaxies: Seyfert - X-rays: galaxies

\section{Introduction}

The fluorescent $\mathrm{K} \alpha$ iron line is an important feature in the high energy spectra of Seyfert galaxies. It is believed to be the result of fluorescent emission due to photoionization of iron atoms in optically thick matter illuminated by a compact source of hard X-ray photons. The study of the iron line properties (ionization state, profile, variability) indeed provides very useful information on the geometry and the physical properties of the emitting material (Fabian et al. 2000 and references therein).

Rapid variability (on less than a day time scale) of the iron line has been reported in the past for a few objects (Yaqoob et al. 1996; Vaughan \& Edelson 2001; Wang et al. 2001). In all these cases, the line was relatively broad. This broadening is believed to be a direct probe of Doppler and gravitational effects that occur in the region very close to the putative supermassive black hole (hereafter $\mathrm{BH}$ ). The line variability would thus be associated with the strong perturbations of the emitting medium in the vicinity of the $\mathrm{BH}$. Moreover, a narrow iron line appears to be often, if not always, present in Seyfert 1 galaxies (Pounds \& Reeves 2002 and references therein). It is thought to be produced by an obscuring and remote torus postulated to exist in

Send offprint requests to: P. O. Petrucci, e-mail: petrucci@obs.ujf-grenoble.fr
AGN unification schemes (Antonucci 1993; Ghisellini et al. 1994). This line is thus not expected to vary on so short time scale.

Mkn 841 is a bright Seyfert 1 galaxy $(z=0.0365)$, one of the rare Seyfert 1 detected by OSSE at more than $3 \sigma$ (Johnson 1997). It is known for its large spectral variability (George et al. 1993; Nandra et al. 1995), its strong soft excess (this was the first object where a soft excess was observed, Arnaud et al. 1985) and its variable iron line (at least on a year time scale, George et al. 1993). The latter was observed in some cases with a relatively large equivalent width (hereafter $E W$ ) of about $400 \mathrm{eV}$ (Day et al. 1990; Bianchi et al. 2001) significantly above the value predicted by standard cold reflection model (e.g. George \& Fabian 1991).

In this paper we present the results from a XMMNewton observation of Mkn 841. This source was also observed simultaneously with the BeppoSAX satellite. We report the presence of a rapidly variable narrow (i.e. unresolved by the XMM-pn instrument) iron line changing by a factor of a few in flux on a half day time scale.

\section{Observation and data analysis}

Due to operational contingency, the $30 \mathrm{ks}$ observation of Mkn 841 with XMM (Jansen et al. 2001 and references therein) was split into two parts, planned the 13th (noted 


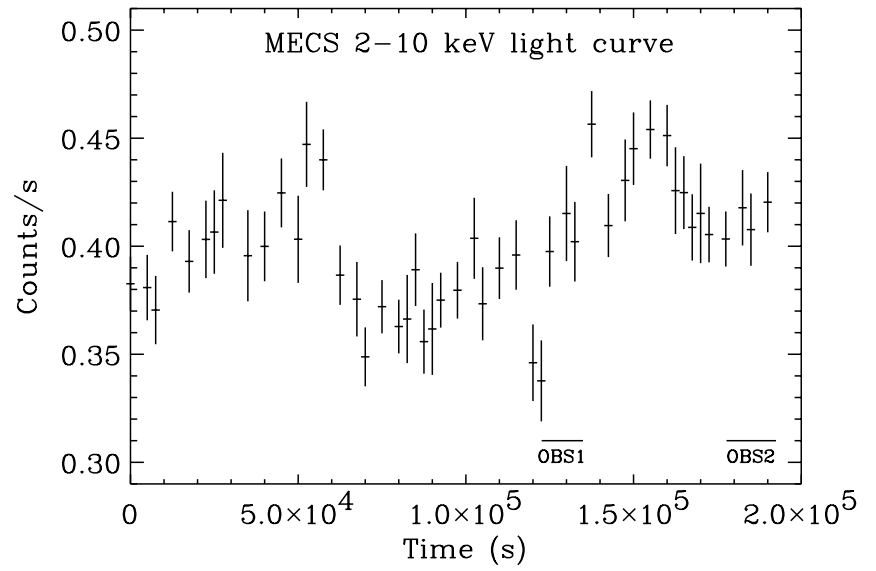

Fig. 1. MECS light curves (bins of $2500 \mathrm{~s}$ ). The periods of the two XMM observations are indicated on the figure.

OBS1) and the 14th (noted OBS2) of February 2001 with $\sim 12$ and 15 ks exposure time respectively. The two observations were separated by about 15 hours.

In this paper, we will only deal with the EPIC-pn data (we have checked that the pn and MOS spectra are consistent with each other in the $2-10 \mathrm{keV}$ band). A complete analysis, including all the XMM instruments, is deferred to a future work. The EPIC-pn camera was operated in Small Window mode (hereafter SW), with thin aluminium filters to reject visible light. The EPIC-pn event file was reprocessed from the ODF data files using the epchain pipeline task of the XMM Science Analysis System (SASv5.2) and using the most updated version of the public calibration files. We note that SASv5.2 only contained a preliminary model for the pn-chips charge transfer efficiency. This should not affect our main results presented here, based on the difference between two spectra, but may change slightly the spectral shape. The source spectra and light curves were built from photons detected within a 40 arcsec extraction window centered on the source. $\mathrm{X}$-ray events corresponding to pattern $\leq 4$ were selected. The background was estimated from an offset position and was found to be extremely low during the whole observation. The EPIC-pn count rate was of about $18 \mathrm{cts} \mathrm{s}^{-1}$ for both observations, i.e. well below the pile-up threshold of $\sim 300 \mathrm{cts} \mathrm{s}^{-1}$ in SW mode. For the fitting procedure the XMM spectra were binned to have at least 50 counts per bin.

Mkn 841 was also observed between the 11th and 14th of February 2001 by the BeppoSAX satellite (Boella et al. 1997 and references therein). The source was pointed for a total net time exposure of $\sim 90,40$ and $20 \mathrm{ks}$ for the MECS, PDS and LECS instruments respectively. Since we focus our analysis, in this paper, to data above $2 \mathrm{keV}$, we will not deal with the LECS data any longer. The MECS light curve is reported in Fig. 1 with a binning of $2500 \mathrm{~s}$. Variations of about $20 \%$ are observed. The periods of the two simultaneous XMM observations are also reported in this figure. The EPIC-pn light curves show smooth flux variations of a few percent on time scale of $1000 \mathrm{~s}$ or less.
The integrated XMM-Newton flux of Mkn 841 over the 2-10 keV range was roughly the same for the two EPIC-pn observations at about $1.4 \times 10^{-11} \mathrm{erg} \mathrm{s}^{-1} \mathrm{~cm}^{-2}$. It slightly differs by $\sim 10 \%$ (smaller) than the mean $2-10 \mathrm{keV}$ flux measured in the MECS as it is generally observed between these two instruments (Molendi private communication).

The $(2-5 \mathrm{keV}) /(5-10 \mathrm{keV})$ hardness ratio of the MECS being consistent with no spectral variability during the observation, and in order to maximize the statistics, we integrated the MECS and PDS spectrum over the entire observation despite only a part of it was simultaneous with the XMM one. In the following, all errors refer to $90 \%$ confidence level for 1 interesting parameter $\left(\Delta \chi^{2}=2.7\right)$.

\section{The line variability}

The XMM-Newton and BeppoSAX instruments need to be carefully normalized one with each other to correct from calibration uncertainties. The MECS data were used to perform the cross calibration as follows. The multiplicative factor between the EPIC-pn and the PDS was obtained by normalizing the EPIC-pn flux to the MECS spectrum and by applying a relative normalization factor of 0.86 (free to vary by $\pm 5 \%$ ) corresponding to the cross calibration of the MECS and PDS instruments (Fiore et al. 1999). We then obtain a normalization factor of 1.13 and 1.08 for the PDS spectrum relative to the EPIC-pn one for OBS1 and OBS2 respectively. From now, we will only use the EPIC-pn and PDS instruments after having checked that the EPIC-pn and MECS spectral shape were consistent with each other in the $2-10 \mathrm{keV}$ band.

We have reported in Fig. 2 the ratio between the EPICpn spectra of OBS1 and OBS2 and the best fit PEXRAV model of XSPEC (Magdziarz \& Zdziarski 1995). This best fit was obtained fitting the EPIC-pn and PDS data simultaneously. A line is clearly visible near $6.2 \mathrm{keV}(\sim 6.4 \mathrm{keV}$ in source rest frame) in OBS1. Indeed the addition of a Gaussian in the fit of OBS 1 is significant at more than $99 \%$ (following the F-test) with $\Delta \chi^{2}=20$ for 3 additional parameters. On the contrary, the addition of a line was not significant in OBS2 $\left(\Delta \chi^{2}=1\right)$. The best fit parameters of the PEXRAV+GAUSSIAN fits are reported in Table 1 .

The energy of the line detected in OBS1 is consistent with a neutral $\mathrm{K} \alpha$ line $E_{\text {line }}=6.41_{-0.06}^{+0.05} \mathrm{keV}$ (source frame), it is unresolved with a width $\sigma_{\text {line }}<170 \mathrm{eV}$ and has an equivalent width $(E W)$ of $120_{-40}^{+50} \mathrm{eV}$ (fitting with a DISKLINE model (Fabian et al. 1989), the data still constrain the line to have a narrow profile, the inner disk radius being larger than $\sim 200$ Schwarzschild radii). We obtain an upper limit of $\sim 70 \mathrm{eV}$ for the line $E W$ in OBS2 and the best fit line flux is a factor $\sim 3$ weaker than in OBS1.

We then checked for line flux variations on smaller time scales. For that purpose, we studied the light curves of the flux ratios $(6-6.6 \mathrm{keV}) /(3-5 \mathrm{keV})$ and $(6-6.6 \mathrm{keV}) /(7-$ $10 \mathrm{keV})$. These ratios are expected to be sensitive to the line flux variations with respect to the continuum shape in each side of the line. The line interval is smaller than the 

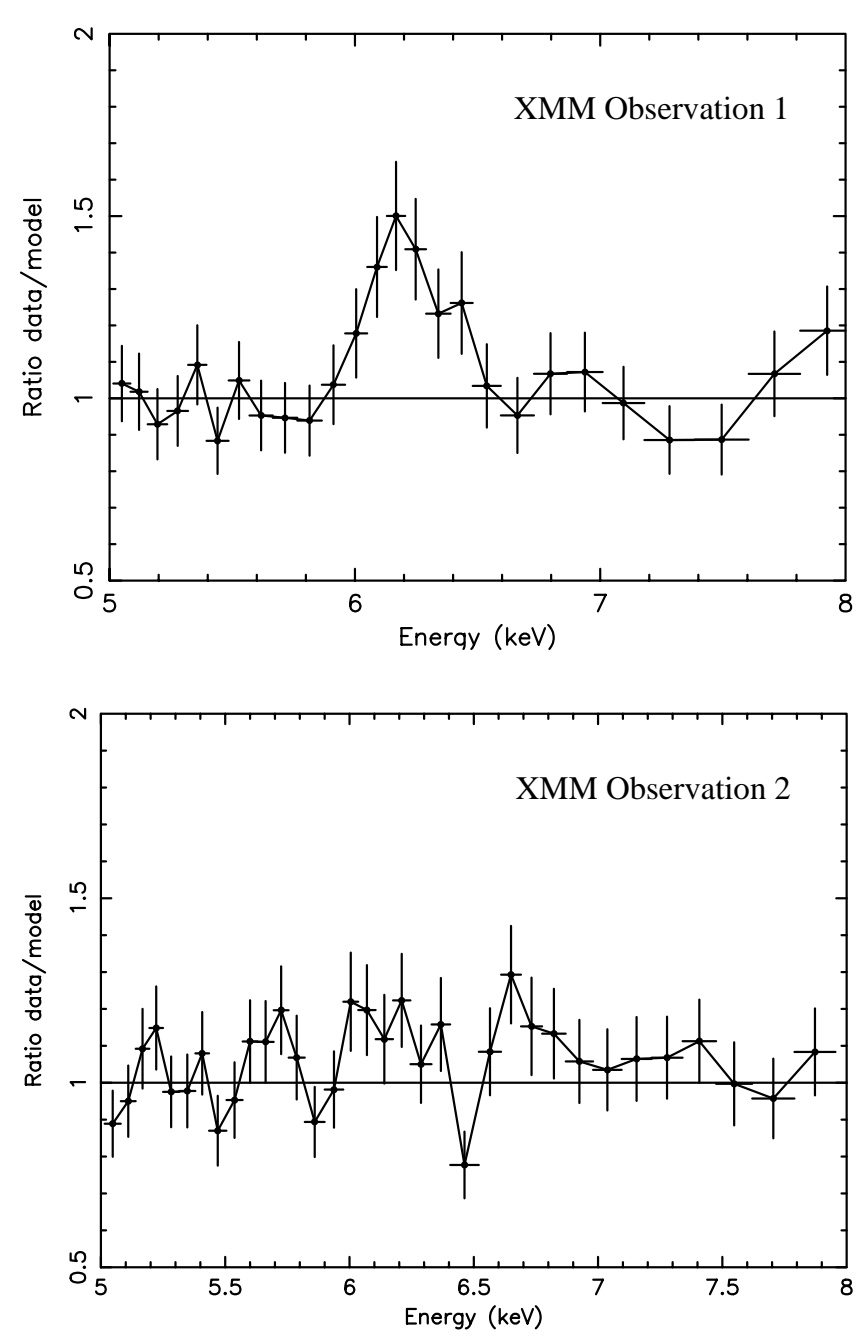

Fig. 2. Ratios data/model (without line) for OBS1 and OBS2. The PEXrAv fit was done using the EPIC-pn and PDS data simultaneously. The vertical dashed line indicates the position of the $6.4 \mathrm{keV}$ neutral iron line in the source rest frame.

Table 1. Best fit parameters for OBS1 and OBS2 when fitting with the PEXRAV+GAUSSIAN model above $2 \mathrm{keV}$. The line energy is in the source rest frame.

\begin{tabular}{ccc}
\hline \hline Observation & OBS1 & OBS2 \\
\hline$\Gamma$ & $2.02_{-0.04}^{+0.06}$ & $1.99_{-0.09}^{+0.10}$ \\
$R$ & $2.1_{-0.9}^{+0.4}$ & $2.0_{-1.0}^{+2.7}$ \\
$E_{\mathrm{c}}(\mathrm{keV})$ & $120_{-50}^{+120}$ & $90_{-40}^{+180}$ \\
$E_{\text {line }}(\mathrm{keV})$ & $6.41_{-0.06}^{+0.05}$ & $6.4^{*}$ \\
$\sigma_{\text {line }}(\mathrm{keV})$ & $<0.17$ & $0.1($ fixed $)$ \\
$F_{\text {line }}\left(10^{-6} \mathrm{ph} \mathrm{cm}{ }^{-2} \mathrm{~s}^{-1}\right)$ & $18_{-6}^{+7}$ & $<11$ \\
$\mathrm{EW}(\mathrm{eV})$ & $120_{-40}^{+50}$ & $<70$ \\
$\chi^{2} /$ d.o.f. & $208 / 243$ & $328 / 312$ \\
\hline
\end{tabular}

* Forced to be in the $6-7 \mathrm{keV}$ energy range.

others to be sensible enough to the line flux variability. The light curves show a significant increase of both flux ratios during OBS1 where the line flux reaches a maximum of $\sim 5 \times 10^{-5} \mathrm{ph} \mathrm{cm}^{-2} \mathrm{~s}^{-1}$. Fits of the different EPIC-pn spectra along the light curves (using a temporal bining of

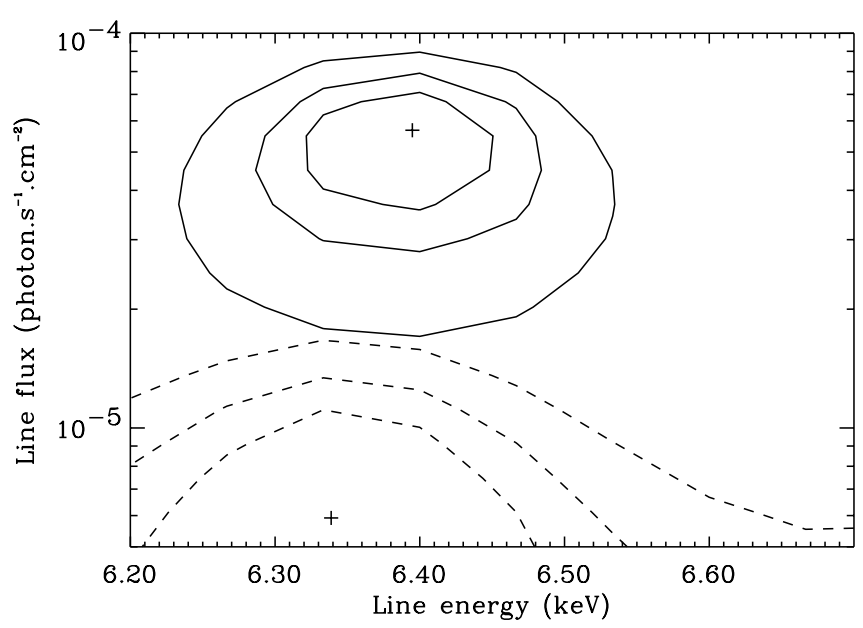

Fig. 3. Contour plot (68\%, $90 \%$ and $99 \%$ confidence level) of the line flux vs. the line energy during the line flux maximum of OBS1 (solid line) and the total OBS2 (dashed line). Cf. text for details.

$1800 \mathrm{~s})$ show that the best fit line flux varies by a factor $\sim 6$ during the total observation (the lowest flux being in OBS2). The line width is always consistent with zero i.e. the line is always unresolved by the instrument.

We have plotted in Fig. 3 the contour plots of the line flux versus the line energy (with contours at 68, 90 and $99 \%$ ) obtained during the sub-interval of OBS1 where the line flux reaches its maximum (solid contours) and for the total OBS2 (dashed contours). These contour plots have been obtained using the EPIC-pn data alone fitted with PEXRAV+GAUSSIAN. The line width was kept fixed to the value of $0.1 \mathrm{keV}$ and we also fixed the reflection normalization parameter $R$ to its best fit value. From these contours, the hypothesis of the constancy of the line can be rejected at more than $99.9 \%$ (it is rejected at $99.5 \%$ if we use the total OBS1). Unfortunately, the BeppoSAX/PDS data does not provide enough counts to test whether the reflection also varied significantly during the pointing.

\section{Discussion}

Two observational facts of the present data are not easy to account for. Firstly the narrow line varies rapidly by a factor of a few while the continuum changes by only $\sim 10-20 \%$. Secondly the reflection seems relatively large, $R>1$, while the line $E W$ is relatively small $<170 \mathrm{eV}$ (it is worth noting that fitting the BeppoSAX data above $2 \mathrm{keV}$ gives also a large $R=2.5_{-1.3}^{+2.5}$ and a small $E W<100 \mathrm{eV}$ ). These points are in contradiction with the standard cold reflection model. In this model, the line width is due to the motion of the emitting matter in the $\mathrm{BH}$ gravitational potential. In this case, a fluorescent neutral iron line with a width of about $150 \mathrm{eV}\left(\sim 14000 \mathrm{~km} \mathrm{~s}^{-1}\right)$, like in our case, is expected to be emitted at a distance $r \simeq 10^{17} M_{8} / q \mathrm{~cm}$ where the BH mass $M_{\mathrm{bh}}=10^{8} M_{8}$ solar masses and $q$ depends on the distribution of the orbital shape and inclination of the matter spiraling around the $\mathrm{BH}$ ( $q$ is expected to be larger than 1, Krolik 2001). 
On the other hand, a variability time scale of $\sim 10$ hours constrains the size $d$ of the line emitting region to about 10 light hours, i.e. $d \simeq 10^{15} \mathrm{~cm}$. The solid angle sustained by the emitting region, as seen by the X-ray source, is then $\Delta \Omega \simeq \pi d^{2} / r^{2} \simeq 3 \times 10^{-4} q^{2} M_{8}^{-2}$. The observed values of $R=\Delta \Omega / 2 \pi>1$ then imply $q M_{8}^{-1} \gtrsim 140$. However, the fact that we do not observe any flare in the MECS light curve, which would be at the origin of the line variability, constrains $r$ to be larger than about 1 light day, meaning $q M_{8}^{-1} \lesssim 40$ in contradiction with the previous estimation and independently of the BH mass. Modifications of this standard view are thus clearly needed.

It is first possible that the observed line variability is not controlled by the continuum itself but by an intrinsic variability of the reflecting material due to, e.g. thermal instabilities. These instabilities may change the ionization state of the upper layers of the matter. The strength of the neutral iron line, expected to be produced in the deeper layers, could then be degraded by the skin scattering properties (Nayakshin \& Kallman 2001; Petrucci et al. 2001). On the other hand, Nayakshin \& Kazanas (2002) also showed that, in response to changes in the X-ray flux, the time for re-adjustment of the hydrostatic balance of the illuminated gas may be longer than the light-travel time between the primary X-ray source and the reflecting material. The line variability observed here may then result from an X-ray flare produced in the inner disc region but occuring some time before the start of the XMM and even BeppoSAX observations. We note however that the change of ionization state of the emitting region suggested above would probably produce a ionized iron line which is not detected.

Alternatively, the line emission may have been affected by the continuum outside of the observed band pass. Variations of the high-energy cut-off and/or of the UVEUV emission can indeed alter the ionization structure of the disc surface without obvious changes in the $2-10 \mathrm{keV}$ continuum (Vaughan \& Edelson 2001). The EPIC data down to $\sim 0.5 \mathrm{keV}$ do not show significant variation between OBS1 and OBS2 but a more precise study of the RGS and OM data will help to better test this hypothesis.

Then, if we admit that the line variability can exist without significant variations of the continuum in the XMM energy range, the distance $r$ of the emitting line region estimated above could be compatible with a half day variability time scale (or less) if the BH mass of Mkn 841 is relatively small, $<10^{6}$ solar masses. It is however significantly smaller than the $\sim 10^{8}$ solar masses recently estimated by Laor (2001).

Interestingly, a narrow profile is also expected, independently of the $\mathrm{BH}$ mass, if the flare at the origin of the line variability occurs in the inner regions but close to the disc and thus illuminates a small fraction of its surface (Nayakshin \& Kazanas 2001; Yaqoob 2001). However, in this case the line is generally expected to be shifted due to relativistic effects, which is not observed.

A less "standard" explanation could be the crossing of an obscuring thick cloud in between the X-ray source and the reflecting matter. This cloud needs to be close enough to the X-ray source to rapidly eclipse a large part of its emission. It can explain why the continuum does not change between the two XMM observations since the cloud may not cross the line of sight. We then would expect the reflection hump to vary, an hypothesis which is unfortunately not testable with the present data.

\section{Conclusion}

The neutral narrow iron line of Mkn 841 appears to be highly variable on a timescale of 10 hours or less. This cannot be easily explained in the standard cold reflection model framework. None of the explanations proposed here appear completely conclusive. A consistent interpretation of these results has to concile the presence of this variable narrow and apparently neutral line, a constant (in flux and shape) underlying X-ray continuum and a large $(>1)$ reflection normalization. New data are clearly needed to confirm this observation and to understand the origin of the line variability.

Acknowledgements. We thanks the referee, S. Vaughan, for his useful comments.

\section{References}

Antonucci, R. 1993, ARA\&A, 31, 473

Arnaud, K. A., Branduardi-Raymont, G., Culhane, J. L., et al. 1985, MNRAS, 217, 105

Bianchi, S., Matt, G., Haardt, F., et al. 2001, A\&A, 376, 77

Boella, G., Chiappetti, L., Conti, G., et al. 1997, A\&AS, 122, 327

Day, C. S. R., Fabian, A. C., George, I. M., \& Kunieda, H. 1990, MNRAS, 247, 15P

Fabian, A. C., Rees, M. J., Stella, L., \& White, N. E. 1989, MNRAS, 238, 729

Fabian, A. C., Iwasawa, K., Reynolds, C. S., \& Young, A. J. 2000, PASP, 112, 1145

Fiore, F., Guainazzi, M., \& Grandi, P. 1999, SAXabc, vs. 1.2, Cookbook for BeppoSAX NFI Spectral Analysis

George, I. M., \& Fabian, A. C. 1991, MNRAS, 249, 352

George, I. M., Nandra, K., Fabian, A. C., et al. 1993, MNRAS, 260,111

Ghisellini, G., Haardt, F., \& Matt, G. 1994, MNRAS, 267, 743

Jansen, F., Lumb, D., Altieri, B., et al. 2001, A\&A, 365, L1

Johnson, N. 1997, BAAS, 190, 2005

Krolik, J. H. 2001, ApJ, 551, 72

Laor, A. 2001, ApJ, 553, 677

Magdziarz, P., \& Zdziarski, A. A. 1995, MNRAS, 273, 837

Nandra, K., Turner, T. J., George, I. M., et al. 1995, MNRAS, 273,85

Nayakshin, S., \& Kallman, T. R. 2001, ApJ, 546, 406

Nayakshin, S., \& Kazanas, D. 2002, ApJ, 567, 85

Nayakshin, S., \& Kazanas, D. 2001a, ApJL, 553, L141

Petrucci, P., Merloni, A., Fabian, A., Haardt, F., \& Gallo, E. 2001, MNRAS, 328, 501

Pounds, K., \& Reeves, J. 2002 [astro-ph/0201436]

Vaughan, S., \& Edelson, R. 2001, ApJ, 548, 694

Wang, J., Wang, T., \& Zhou, Y. 2001, ApJ, 549, 891

Yaqoob, T., Serlemitsos, P. J., Turner, T. J., George, I. M., \& Nandra, K. 1996, ApJ, 470, L27

Yaqoob, T. 2001, Proc. of New Century of X-ray Astronomy, Japan, ed. H. Inoue, \& H. Kunieda, ASP Conf. Ser. 